Ana Seradilla Castaño

Universidad Autónoma de Madrid

\title{
IR Y CAER COMO CONSTITUYENTES DE LOCUCIONES FRASEOLÓGICAS QUE NO IMPLICAN MOVIMIENTO'
}

1. En todas las lenguas se cuenta con un importante número de expresiones idiomáticas que han de ser analizadas como lexías globales, en el sentido de que su significado es unitario y no la suma de significados de sus partes. En este trabajo he decidido centrarme únicamente en locuciones formadas por los verbos ir y caer más un sustantivo, que va, generalmente, precedido de preposición. Más concretamente, he limitado mi estudio a los casos en los que estas locuciones no expresan movimiento sino que hacen referencia a un proceso psicológico o a una situación que no trae consigo ningún desplazamiento real, pese a que el verbo base es, claramente, un verbo de movimiento.

El objetivo de este estudio es, tras establecer un corpus inicial, delimitar el tipo de construcciones que forman parte de nuestro análisis, realizar la comparación con otras expresiones equivalentes en inglés y comprobar, por una parte, si estamos ante el mismo tipo de estructura léxico-sintáctica y, por otra, si para expresar los mismos significados se recurre en esta lengua también a verbos de movimiento o si, por el contrario, se recurre a verbos que denotan estado. Una vez establecida la comparación, se analizarán los valores que los verbos de movimiento aportan, la razón de la utilización de determinadas metáforas y se intentará responder a la pregunta de por qué, en ocasiones, ambas lenguas se valen de estos verbos con sentido metafórico para crear expresiones de este tipo y por qué en otros casos es sólo el español el que se vale de estas construcciones.

2. El corpus con el que trabajaré es relativamente restringido ya que he limitado el campo de estudio exclusivamente a aquellos casos en los que, como ya he señalado, los verbos ir y caer no implican un movimiento físico²:

\begin{tabular}{|l|l|}
\hline CAER CHUZOS DE PUNTA & CAER DE PIE \\
\hline CAER EN DESGRACIA & CAER EN LA CUENTA \\
\hline CAER EN LA RED & CAER EN LA TENTACIÓN \\
\hline CAER EN SACO ROTO & NO CAER ESA BREVA \\
\hline CAER POR SU PROPIO PESO & CAÉRSELE (A ALGUIEN) EL ALMA A LOS PIES \\
\hline CAÉRSELE (A ALGUIEN) EL PELO & CAÉRSELE (A ALGUIEN) LA BABA \\
\hline
\end{tabular}

${ }^{1}$ La propuesta que presento en este artículo forma parte de un proyecto de investigación más amplio: «Las expresiones idiomáticas con verbos de movimiento. Propuesta de elaboración de un diccionario teórico, de uso y contrastivo». Dicho proyecto, dirigido por la Dra. Elena de Miguel, ha sido financiado por la UAM dentro de su programa de ayudas a grupos precompetitivos. Una primera versión de este estudio fue presentada en el VII Congreso de la Mediterranean Studies Association (Universidad de Barcelona: mayo de 2004).

2 Expresiones como éstas son de larga tradición en nuestra lengua. Valgan como muestra algunas de las incluidas en el Tesoro de Covarrubias (1611): caer chuzos de punta - se usaba en español clásico una metáfora casi idéntica: caer del cielo lanzas; caer de pie - se usaba la variante caer de pies como gato; caer en desgracia (caer de su gracia); caer en la cuenta; caer en la red; irse de la lengua; irse por los cerros de Úbeda; ir viento en popa. Dice además el autor: «Otras fórmulas hay del verbo ir; déjolas por no cansar», lo que nos permite pensar que, aunque no aparezcan en este diccionario, seguramente había otras muchas expresiones con este verbo. 


\begin{tabular}{|l|l|}
\hline CAÉRSELE (A ALGUIEN) LA CARA DE VERGÜENZA & CAÉRSELE (A ALGUIEN) LA CASA ENCIMA \\
\hline CAÉRSELE (A ALGUIEN) LOS ANILLOS & \\
\hline IR A LA DERIVA & NO IR A LA ZAGA \\
\hline IR A LO SUYO & IR A POR UVAS \\
\hline IR A TIRO HECHO & IR AL GRANO \\
\hline IR (ALGO) A MISA & IR CON PIES DE PLOMO \\
\hline IR CONTRA CORRIENTE & IR DE CABEZA \\
\hline IR DE CRÁNEO & IR DE CULO \\
\hline IR DE GUATEMALA A GUATEPEOR & IR DE HERODES A PILATOS \\
\hline IR DE MAL EN PEOR & IR DE PUNTA EN BLANCO \\
\hline IR DE TIROS LARGOS & IR DE TRAPILLO \\
\hline IR HECHO UN ADÁN & IR HECHO UN GITANO \\
\hline IR HECHO UN PINCEL & IR MUY PUESTO \\
\hline IR QUE CHUTA & IR SOBRE RUEDAS \\
\hline IR VIENTO EN POPA & IRSE A PIQUE \\
\hline IRSE AL GARETE & IRSE DE LA LENGUA \\
\hline IRSE DE ROSITAS & IRSE (A ALGUIEN) EL SANTO AL CIELO \\
\hline IRSE (A ALGUIEN) LA FUERZA POR LA BOCA & IRSE POR LAS RAMAS \\
\hline IRSE POR LOS CERROS DE ÚBEDA ${ }^{3}$ & \\
\hline
\end{tabular}

3. Se hace necesario, a la vista del corpus propuesto, delimitar con claridad el tipo de expresiones ante las que nos encontramos. Lewis (2002) habla de una serie de unidades léxicas graduadas de mayor a menor fijación o cohesión: palabra, frase hecha, colocación y expresión institucionalizada $a^{4}$ En este estudio me limitaré al análisis de determinadas frases hechas o locuciones ${ }^{5}$, diferentes de las colocaciones; este dato es importante pues va a ser decisivo a la hora de interpretar el funcionamiento y el significado de los verbos que las integran. Si seguimos las pruebas que Kazumi Koike (2001) presenta en su obra para diferenciar colocaciones y locuciones, observamos que, efectivamente, estamos ante este último tipo de estructuras, que presentan un importante grado de cohesión ${ }^{6}$.

${ }^{3}$ He dejado de lado en mi estudio locuciones como irse a freír espárragos, irse al infierno, irse al cuerno, etc, que también en inglés se expresan con un verbo de movimiento - to go jump in the lake, to go to hell-. La razón de su exclusión es que, aunque el sentido metafórico es claro, presentan una construcción diferente ya que suelen aparecer siempre con el verbo en forma imperativa - es el hablante el que exige al oyente un movimiento real o figurado - y requieren un análisis en mayor profundidad, que en este momento es imposible por razones de espacio.

${ }^{4}$ Se trata, según Corpas Pastor (2003: 148), de una situación universal ya que en todas las lenguas encontramos fórmulas fraseológicas tales como colocaciones: fr. gravement malade; locuciones: esp. venir como anillo al dedo; paremias: al. der Apfel fällt nicht weit vom Stamm ('de tal palo tal astilla') y fórmulas o expresiones institucionalizadas: esp. ni hablar del peluquín. Como ya he señalado, este estudio se va a ceñir al análisis de locuciones exclusivamente.

${ }^{5}$ Véase, en este sentido, la diferencia entre estas locuciones y aquellas otras en las que ir y caer no forman estas expresiones sino simples combinaciones sintagmáticas: ir a casa, ir de excursión, caer en la cuneta, caer de rodillas.

${ }^{6}$ En los apéndices que este autor presenta no existe, de hecho, ni un solo caso en los que ir o caer aparezcan en colocaciones. 
Koike, que recoge en su trabajo las diferentes pruebas utilizadas por otros autores, señala, en primer lugar, que la colocación tolera la modificación adjetival, mientras que la locución no lo permite. En el corpus que manejamos, esto se hace evidente; así, frente a hacer un aterrizaje/ hacer un aterrizaje forzoso (Koike 2001: 31), no es posible decir:

\author{
*caer chuzos de punta gruesa \\ *caer en desgracia terrible \\ *caérsele a alguien el pelo largo \\ *ir a por uvas verdes \\ *ir hecho un gitano elegante \\ *irse de rositas blancas \\ *irse la fuerza por la boca grande
}

Por otra parte, menciona la relativización, es decir, la capacidad del sustantivo para transformarse en el antecedente de la cláusula relativa, posible sólo en las colocaciones:

Este libro marca la línea que deben seguir sus partidarios (colocación: seguir la línea; Koike: p. 32).

*Los anillos que no se le caen a María por fregar los platos

* El santo al cielo que se le fue a Pilar cuando vio pasar a Juan

Obsérvese, sin embargo, que en algunos casos, esta operación, aunque extraña, es también posible en algunas de las frases hechas con las que estamos trabajando; en estos casos se percibe una menor cohesión sintagmática y, al menos, en el primer ejemplo mostrado parece que nos encontramos ante un sentido más literal que idiomático:

Era difícil salir de la red en la que cayó.

La tentación en la que caíste era imposible de evitar.

Se menciona también en esta obra la posibilidad de nominalización del verbo en la colocación; posibilidad que, según este autor, es mínima en el caso de las locuciones. A la vista de los datos con los que contamos, podemos concluir que, efectivamente, esta nominalización ocurre muy extrañamente:

? La caída de chuzos de punta provocó varios accidentes

? Su ida por las ramas desconcertó al auditorio

No pude evitar la caída en la tentación

Comenta también Koike cómo en las colocaciones es posible la extracción de un componente, mientras que las locuciones no suelen permitir esta posibilidad; en nuestro caso, efectivamente, no podemos extraer un elemento a no ser que se utilice con su sentido literal:

Se te va a caer el pelo, ese pelo tan largo que tienes, si no haces lo que digo.

\footnotetext{
${ }^{7}$ No quiere decir esto que no acepten nunca modificadores; véase en este sentido: caer en la red tejida / urdida por alguien; caer en la tentación de comer; no ir a la zaga de alguien. Al fin y al cabo, estamos ante fórmulas no ante palabras, que son las que presentan el máximo grado de cohesión.
} 
Relacionada con esta prueba, está la de la eliminación (o adición) que propone Corpas (1997). Según esta autora si añadimos o eliminamos un elemento de la locución, el resultado puede ser gramatical pero perderá su carácter idiomático: *matar pájaros de un tiro /*matar dos pájaros blancos de un tiro (Corpas 1997: 90).

* Se va el santo

*No se te van a caer los anillos al fregadero por fregar los platos

Por último, la flexibilidad morfológica de las colocaciones permite transformaciones que no son frecuentes en las locuciones:

trabar amistad > muchas amistades, amistad trabada (colocación) (Koike: 33)

irse el santo al cielo $>$ *los santos al cielo, *el santo a los cielos

Esto no quiere decir que en las frases hechas no exista la posibilidad de algunas transformaciones morfosintácticas:

caer en la red-caer en las redes

caer en la (una) tentación

ir a lo suyo - ir a lo mío - ir a lo tuyo

Entre las locuciones que analizamos, algunas, incluso, permiten cambiar el verbo de movimiento por otro verbo sin que el significado de la locución varíe:

Ir con pies de plomo $=$ andar con pies de plomo

Ir de cabeza = andar de cabeza

Estos cambios, poco frecuentes en las expresiones que componen nuestro corpus, no son, sin embargo, extraños en las frases hechas en general, incluso entre verbos de cariz muy diferente; véase el caso de andar a la greña - estar a la greña, donde el hecho de usar un verbo de movimiento o un verbo de estado no afecta al significado global de la expresión. Hay ocasiones en las que sí se da un cambio de significado: caer en saco roto - echar en saco roto pero esta diferencia significativa viene determinada por las características del verbo y el sujeto que admite; así, en el primer caso 'algo cae en saco roto', mientras que en el segundo 'alguien echa algo en saco roto'; en todo caso, el significado del sintagma preposicional permanece inmutable. En los ejemplos de nuestro corpus no encontramos, de todas formas, más casos en los que se dé esta posibilidad ${ }^{8}$. Además, mientras en otras combinaciones de palabras la alteración del orden es posible, en el caso de las locuciones, la reordenación de los elementos integrantes (Corpas 1997: 90) no parece posible: *dar liebre por gato. Puede comprobarse lo que acabamos de decir en los ejemplos con los que estamos trabajando: *ir de guatepeor a Guatemala, *ir de Pilatos a Herodes...

Ante los datos obtenidos, podemos concluir con Koike y Corpas que la fijación estructural es casi total en la locución y que ésta presenta menor flexibilidad formal que la colocación.

8 También señala Koike cómo la pronominalización del sustantivo que actúa como C.D. se da en las colocaciones, pero sólo de forma esporádica en las locuciones. Es lo mismo que ocurre con la posibilidad de transformación en pasiva. Dado que nuestro corpus está formado por verbos intransitivos, esta prueba resulta irrelevante. 
4. Tengo que aclarar, por otra parte, que, aunque estamos ante locuciones fraseológicas, como ya se ha visto gracias a las pruebas empleadas, éstas pueden presentar una estructura sintáctica diferente; así, tenemos casos de

Verbo + sujeto: caer chuzos de punta; (no) caer esa breva

Verbo + sujeto + C. I. + (C. C.): caérsele (a alguien) el alma a los pies; caérsele (a alguien) el pelo; caérsele (a alguien) la baba; caérsele (a alguien) la cara de vergüenza; caérsele (a alguien) la casa encima; caérsele (a alguien) los anillos, írsele (a alguien) el santo al cielo; írsele (a alguien) la fuerza por la boca ${ }^{9}$

Verbo + C. C. (Prep.): caer de pie; caer en desgracia; caer en la cuenta; caer en la red; caer en la tentación; caer en saco roto; ir a la deriva; no ir a la zaga; ir a lo suyo; ir a por uvas; ir a tiro hecho; ir al grano; ir (algo) a misa; ir con pies de plomo; ir contra corriente; ir de cabeza; ir de cráneo; ir de culo; ir de punta en blanco; ir de tiros largos; ir de trapillo; ir sobre ruedas; irse a pique; irse de la lengua; irse de rositas; irse por las ramas; irse por los cerros de Úbeda; ir que chuta; ir viento en popa

Verbo + C. Direcc. + C. Direcc.: ir de Guatemala a Guatepeor; ir de Herodes a Pilatos; ir de mal en peor

Verbo + C. Pred.: ir hecho un adán; ir hecho un gitano; ir hecho un pincel; ir muy puesto

No obstante, esta diferente estructura sintáctica no resulta ahora relevante para nuestro análisis, ya que no lleva consigo ninguna diferencia en el significado de los verbos que forman parte de las locuciones analizadas ${ }^{10}$.

5. En cuanto a la semántica, en la que a partir de ahora nos vamos a detener, hay un aspecto fundamental que me gustaría señalar. Mientras que en las colocaciones el significado es transparente, es decir «el significado de una colocación refleja los significados de sus partes constituyentes (composicionalidad semántica)» (Koike 2001: 35), esto no ocurre en las locuciones; por eso el verbo de las expresiones que estamos analizando puede no indicar movimiento ya que está incluido en una locución de valor global, en la que se difuminan los significados de las partes. Este hecho es básico porque, como decimos, en las colocaciones el verbo mantiene su significado original y sólo en las locuciones sería posible la utilización metafórica que observamos en los ejemplos de nuestro corpus.

Por otra parte, mientras que las colocaciones presentan un solo significado, en las locuciones es frecuente que se den dos significados: el literal y el metafórico. Así, caérsele a alguien la casa encima puede, obviamente, interpretarse de forma literal, al igual que irse a pique o ir a por uvas, pero estos casos ya no nos interesan pues pierden su carácter idiomático.

\footnotetext{
${ }^{9}$ Estas son las que Corpas llama «locuciones clausales» y están «formadas por varios sintagmas, de los cuales, al menos uno es verbal. Están provistas de sujeto y predicado, pero no forman oraciones completas porque necesitan actualizar algún actante en el discurso. Son más abundantes en español, aunque en inglés se registran algunos ejemplos.» (Corpas 2003: 141).

${ }^{10}$ Un análisis sintáctico exhaustivo de este tipo de expresiones, desde una perspectiva generativista, puede leerse en Mendívil (1999).
} 
Nos fijaremos ahora, una vez delimitada la categoría a la que pertenecen las expresiones objeto de estudio, en las metáforas utilizadas. En primer lugar, quiero señalar que no estamos ante metáforas de tipo individual sino ante metáforas reconocidas como tales por la comunidad y recogidas, por tanto, en los diccionarios generales. Por otro lado, parece demostrado que todas las lenguas presentan expresiones idiomáticas del tipo de las que estamos analizando ${ }^{11}$.

A continuación, veremos si es también universal el tipo de metáfora usado. En este sentido, conviene acudir a las reflexiones de Galán (1993: 154). Esta autora nos indica cómo los verbos de movimiento son especialmente productivos para expresar tiempo, sentimientos, ideas, emociones... en las diferentes lenguas y, aunque hay algunas diferencias entre una lengua y otra, ya que «la expresión del movimiento metafórico se construye sobre esquemas sintácticos similares a los utilizados para designar el desplazamiento real» y estos esquemas pueden variar, sí parece claro que «el tránsito del ámbito espacial concreto al ámbito abstracto se realiza mediante esquemas conceptuales compartidos por lenguas no necesariamente emparentadas cultural o históricamente;». Por esta razón encontraremos muchos casos en los que tanto el inglés como el español se valen de estructuras similares y otros en los que se recurre a construcciones diferentes.

5.1. Por lo pronto, el verbo caer, el primero de los que estamos analizando lleva consigo el sentido de 'ir abajo', y en nuestra tradición el concepto 'abajo' tiene un matiz negativo, frente a lo que está arriba, que es positivo. (abajo = malo; arriba = bueno). García Jurado (2000) recoge esta idea y nos informa ${ }^{12}$ de cómo esta metáfora orientacional, que nos permite articular los conceptos a partir de un espacio imaginario definido verticalmente, está ya presente en latín, donde ««Lo ascendente es positivo», mientras que «Lo descendente es negativo»»(p. 1573). Este autor nos demuestra esta realidad a través de expresiones como «Los males caen o sobrevienen» o la metáfora de la lluvia «Caer un chaparrón». Asimismo, y cito literalmente: «Pero no sólo puede caernos un mal, sino que también nosotros podemos caernos en uno o hundirnos en él (...) El abatimiento se expresa tanto en latín como en castellano con esta metáfora descendente de carácter negativo, de donde tenemos el nombre de una enfermedad de moda, 'la depresión'» (2000: 1574).

Basta con echar una ojeada rápida a las expresiones que estamos analizando, para observar que esa idea de «malo, negativo» está presente en casi todas ellas. De hecho, sólo falta en un par de ocasiones; es el caso de caer de pie, que, precisamente, matiza la forma en que se cae y que si se interpretara de forma literal, evidentemente, sería la mejor forma de 'caer', ya que es la que menos riesgos conlleva para quien sufre la caída ${ }^{13}$. El caso de caer en la cuenta es peculiar ya que no hay un matiz negativo, sino que puede entenderse el uso del verbo con preposición como la expresión de la localización de algo, por parte de alguien que ha estado perdido; de hecho, en to get the point no se utiliza la metáfora de la caída sino que hay un matiz positivo, ya que se orienta al futuro: 'obtener, lograr'. En todos los demás casos, como digo, la metáfora espacial que relaciona lo que está abajo con lo negativo está muy presente y por esta razón se recurre a un verbo como caer. Incluso en caer chuzos de punta, donde realmente hay algo que 'cae', y por tanto

\footnotetext{
${ }^{11}$ Makkai (1978), apud Mendívil (1999: 39).

${ }^{12}$ Sigue en este sentido a Lyons (1980: 625).

${ }^{13}$ En inglés to land on one's feet. También aquí hay un verbo de movimiento hacia abajo en el sentido de 'tomar tierra'.
} 
hay un movimiento no figurado, se puede percibir este carácter negativo agudizado por el sujeto metafórico ${ }^{14}$.

Esta metáfora que relaciona lo 'malo' con 'abajo' se da también en inglés, como muy bien señalaron en su momento Lakoff y Johnson (2001: 50-58). Parece que estamos ante una metáfora de carácter universal ${ }^{15}$. En las expresiones que estamos analizando observamos cómo se utiliza una metáfora del mismo sentido en los siguientes ejemplos: (caer en desgracia) to fall from grace; (caer en la red) to fall for; (caer en la tentación) to fall into temptation; (caer en saco roto) to fall on deaf ears; (caérsele a alguien los anillos) to go down in people's eyes ${ }^{16}$.

En otros ejemplos como to stick out like a sore thumb (caer por su propio peso) no se usa el verbo «caer» pero sí se puede percibir cierta idea de movimiento. Dejo de lado una expresión como no caer esa breva, que no tiene una equivalencia clara en inglés ya que not to be lucky no refleja exactamente la estructura a la que nos referimos; creo que estamos ante un caso muy específico del español. En todo caso, en este ejemplo nos encontramos con una fórmula diferente a las demás ya que el sentido figurado lo aporta básicamente el sujeto ${ }^{17}$.

Debemos fijarnos ahora en una serie de expresiones caracterizadas por la presencia de un sujeto y un incremento pronominal dativo ${ }^{18}$ : caérsele a alguien el alma a los pies; caérsele a alguien el pelo; caérsele a alguien la baba; caérsele a alguien la cara de vergüenza; caérsele a alguien la casa encima. En todas ellas se recurre en español a la metáfora orientacional de carácter negativo. Hay un movimiento figurado en dirección descendente. Tenemos que destacar, sin embargo, que en el caso de inglés no se recurre a un verbo de movimiento de carácter figurado sino que en todos esos casos se prefiere un verbo estativo; es más en algunos casos no se recurre a metáforas ${ }^{19}$. La diferencia básica estriba en que, mientras en español se acude a un verbo como caerse, que implica un cambio de estado, y, por lo tanto, se pone el interés en el proceso, en inglés se hace hincapié en el resultado.

5.2. En el caso de $i r$, aunque el verbo implica un desplazamiento de un punto a otro, no está funcionando ninguna metáfora espacial tan clara, por lo que hay expresiones tanto de orientación positiva como de orientación negativa. En las construcciones que nos ocupan no existe un movimiento real pero sí se da, en algunos casos, un desplazamiento

\footnotetext{
${ }^{14}$ Es lo mismo que ocurre en inglés, donde se utiliza el verbo 'llover' pero se recurre a un sujeto también metafórico: to rain cats and dogs. Si tenemos en cuenta las observaciones de Lakoff y Johnson (2001: 54), estamos también ante un sentido metafórico orientacional negativo ya que se suele hacer la identificación «hombre es arriba», «animal es abajo».

${ }^{15}$ Véase cómo incluso la sociedad está organizada en «clases altas» $\mathrm{y}$ «clases bajas».

${ }^{16} \mathrm{El}$ verbo to fall se usa también en expresiones como to fall sound asleep 'quedarse frito', donde también funciona una metáfora orientacional: «lo consciente es arriba; lo inconsciente es abajo» (Lakoff y Johnson: 51); así, en español también decimos cayó dormido, se hundió en un profundo sueño.

${ }^{17}$ De todas formas, también aquí hay un matiz negativo propiciado por la aparición del adverbio de negación ya que «caer esa breva» sería positivo.

${ }^{18}$ Este incremento dativo se correspondería, según Galán (1993: 156) «(como punto final) con el complemento locativo (en caso de que lo hubiera) de los verbos de movimiento real». Compárese, en este sentido: caéersele a alguien la baba - caerse al suelo la baba.

${ }^{19}$ Caérsele a alguien el alma a los pies (to tear someone's heart out, to feel one's heart sink, to have one's world cave in); caérsele a alguien el pelo (to be in trouble, to get it); caérsele a alguien la baba (to slaver at the mouth, to drool); caérsele a alguien la cara de vergáenza (to be ashamed of something); caérsele a alguien la casa encima (to be crushed or overwhelmed with trouble, to have the bottom drop out of one's world).
} 
figurado de pensamientos o sensaciones y ésa es la razón por la que se recurre a este verbo. Señala Galán (1993) que los verbos de movimiento tales como ir presentan en español un carácter mucho más genérico que los verbos alemanes; es posible, pues, que sea este valor genérico el que facilite su entrada en estas expresiones con valor figurado. Dice esta autora: «el español utiliza verbos absolutos (poco orientados) cuyos valores metafóricos se desprenden del contexto o de la estructura de cada construcción.» (Galán 1993: 155). En este sentido, pues, a diferencia de lo que pasa normalmente con las expresiones en las que entra caer, son los otros elementos de la locución los que aportan estos valores y no tanto el verbo, que tiene un carácter más absoluto.

Veamos ahora las equivalencias de estas expresiones en inglés, para poder observar similitudes y diferencias. En primer lugar, señalaré aquellos casos en los que el inglés no recurre a una metáfora con verbos de movimiento:

Para la siguiente serie de expresiones, referidas a la vestimenta o al arreglo personal, el español usa un verbo genérico de movimiento como es $i r$, aunque el sujeto del verbo no realiza necesariamente movimiento alguno, sino que se limita a llevar encima determinadas prendas o a lucir determinado aspecto: el inglés, por su parte, opta por utilizar el verbo 'vestir' o hacer una comparación. Vemos, pues, claramente, cómo el español, frente a otras lenguas, opta por la utilización de un verbo con valor más genérico:

ir de punta en blanco
ir de tiros largos
ir de trapillo
ir hecho un adán
ir hecho un gitano
ir hecho un pincel
ir muy puesto

- to dress up, to dress in full armor

- to dress up, formally

- to dress casual

- to dress carelessly, to look a mess

- to dress untidily, to look a mess

- to dress neatly / nice and neat / stylish, to look smart

- to dress properly, to look smart

En los siguientes casos el inglés tampoco opta por los verbos de movimiento. Parece claro que, a diferencia de caer, que tiene un valor más específico, y que se utiliza con el mismo valor metafórico en diversas lenguas, ir es más usado con este valor de desplazamiento metafórico en español que en otras lenguas y son muchos los casos en los que el inglés no recurre a to go o a otro verbo de movimiento para expresar estas metáforas: ir a la deriva - to drift, no ir a la zaga - to be as good as, to keep up with someone or something, ir a lo suyo - to look after one's own interest, ir a por uvas - to let one's minds wander, to be absent-minded, ir a tiro hecho. En este último caso el inglés no tiene una locución equivalente y se hace necesaria una paráfrasis: to go to get something without hesitation; to go directly to do something. En los siguientes ejemplos nos encontramos también en inglés ante fórmulas que no usan verbos de movimiento: Ir algo a misa - to be the truth/undeniable, ir que chuta - to have enough with something, to be content, irse a pique - to sink, to be ruined, irse de la lengua - to let something slip, to blurt out, irse el santo al cielo - to forget what one was about to say or to do, irse la fuerza por la boca - to brag, to say whatever comes into one's head, to talk a lot of hot air, irse por las ramas - to wander off the point, irse por los cerros de Úbeda - to wander off the point. Obsérvese que estas fórmulas tienen en español importantes diferencias semánticas y presentan estructuras sintácticas muy variadas y, sin embargo, en todas ellas se recurre a la utilización de un mismo verbo que trae consigo la expresión de un movimiento figurado. En 
inglés estas diferencias semánticas y sintácticas se hacen extensibles también a las metáforas usadas, ya que son muy variados los verbos encontrados:

Nos centraremos ahora en una serie de casos en los que sí se usan en inglés verbos de movimiento. Obsérvese, sin embargo, que como estamos diciendo, esta lengua opta con mayor frecuencia por verbos de movimiento más concretos que el genérico to go:

$\begin{array}{ll}\text { ir al grano } & - \text { to come to the point, to get the point } \\ \text { ir con pies de plomo } & - \text { to walk on eggshells } \\ \text { ir contra corriente } & - \text { to swim against the tide }{ }^{20} \\ \text { ir de cabeza } & - \text { to go downhill, to go crazy } \\ \text { ir de cráneo } & - \text { to go downhill, to go crazy } \\ \text { ir de culo } & - \text { to go downhill, to go crazy } \\ \text { ir de Guatemala a Guatepeor } & - \text { to go from bad to worse, to go out of the frying pan into } \\ & \text { the fire } \\ \text { ir de Herodes a Pilatos } & - \text { to go from bad to worse, to go out of the frying pan into } \\ & \text { the fire } \\ \text { ir de mal en peor } & - \text { to go from bad to worse, to go out of the frying pan into } \\ & \text { the fire } \\ \text { ir sobre ruedas } & - \text { to go well } \\ \text { ir viento en popa } & - \text { to go really well / favorable } \\ \text { irse de rositas } & -\end{array}$

Hablábamos respecto a caer de orientaciones negativas o positivas. En el caso de las fórmulas con caer es claramente el verbo el que marca el matiz negativo; en el caso de las formas con $i r$, como ya he avanzado, suelen ser los demás elementos del contexto los que marcan la orientación: irse a pique (el contexto es negativo - 'hundirse'); ir de mal en peor; ir de Herodes a Pilatos. Sólo en unos pocos casos el matiz negativo se indica por el valor de alejamiento que lleva implícito: irse el santo al cielo. No obstante, en estas locuciones no predomina una orientación negativa o positiva ya que, como he dicho, el valor semántico lo aporta el contexto en el que se incluye $i r$, es decir, el resto de la fórmula y no el verbo. Por esta razón hay también muchas locuciones de orientación positiva: ir viento en popa, ir a misa, ir al grano, irse de rositas. Hablamos, pues, de un movimiento figurado pero, dada la naturaleza del verbo implicado, la orientación no depende de éste sino del resto de los elementos implicados, que pueden traer consigo una orientación positiva o negativa.

\section{Conclusiones}

En este trabajo he procedido a identificar, en un primer momento, el tipo de expresiones analizadas a través de la aplicación de una serie de pruebas propuestas por Koike y Corpas, que nos llevan a poder afirmar que estamos evidentemente ante locuciones. Después, a la vista de las diferentes estructuras sintácticas que estas fórmulas presentan, se ha podido comprobar que el hecho de expresarse con una u otra estructura no tiene una repercusión clara en la semántica de estos usos. A continuación, se ha llevado a cabo el

\footnotetext{
${ }^{20}$ Este es un ejemplo claro de cómo el inglés utiliza un verbo más específico mientras que el español puede presentar el mismo valor metafórico con un verbo más absoluto o genérico, tal y como señalaba Galán.

${ }^{21}$ En este caso y en el siguiente vemos cómo en inglés no se recurre a un locución.
} 
análisis del significado de una serie de fórmulas con ir y caer y hemos hecho una comparación con el inglés. De este análisis se deduce que en el caso de caer, que tiene un valor más concreto, hay mayores similitudes entre ambas lenguas; por el contrario, estas son menores con el verbo ir ya que en este caso se observa una característica particular del español, como lengua romance, reseñada por Galán ${ }^{22}$; esta particularidad consiste en que nuestra lengua tiende a usar verbos con un valor absoluto o genérico, cosa que hacen con más dificultad las lenguas sajonas.

Se ha hablado también, a lo largo de este estudio, de orientaciones negativas o positivas. En el caso de las fórmulas con caer es claramente el verbo el que marca el matiz negativo ya que, dentro de nuestra cultura existe desde antiguo una metáfora orientacional que identifica 'abajo = negativo'; en el caso de las formas con ir está metáfora no está presente y suelen ser los demás elementos que componen la locución los que marcan la orientación, que puede ser negativa, como en el caso de irse a pique (el contexto es negativo - 'hundirse'), o positiva: ir viento en popa.

Ante la pregunta de por qué se recurre a verbos como ir o caer en estas perífrasis que no implican ningún movimiento real, podemos responder que estamos ante verbos que presentan una gran facilidad para trasponerse como usos figurados de sentimientos y sensaciones. En este sentido, como afirmaba Galán, el movimiento puede entenderse como un movimiento en el pensamiento ${ }^{23}$.

Con Lakoff y Johnson concluimos, pues, que existen determinadas metáforas universales; así, las orientacionales son relativamente similares en las distintas lenguas. Por otra parte, no podemos perder de vista que hay diferencia entre las distintas lenguas y que las lenguas analizadas se valen de distintos recursos para representar la realidad, por ello, mientras el español se vale con gran facilidad de verbos absolutos o genéricos para expresar el movimiento figurado, otras lenguas como el inglés o el alemán son más reacias a este uso y optan por verbos más concretos para expresar estas metáforas. En todo caso, y aunque no haya coincidencia en todas las locuciones analizadas, se hace evidente que tanto el inglés como el español tienen la posibilidad de recurrir a verbos de movimiento para expresar desplazamientos figurados. Habrá que investigar más las causas profundas de que sea así ya que nos encontramos ante un hecho fundamental en la medida en que tiene una enorme repercusión tanto en la traducción como en la adquisición de segundas lenguas.

\footnotetext{
${ }^{22}$ Algunas, muchas, de estas metáforas se veían ya en latín y están documentadas en nuestra lengua desde antiguo. Véanse en este sentido los ejemplos que hemos citado de Covarrubias.

${ }^{23}$ Esta utilización de verbos de movimiento con un valor figurado se observa también en expresiones idiomáticas del campo de la conversación como ya caigo y otras similares.
} 


\section{BIBLIOGRAFÍA}

Castillo Herrero, $\mathbf{M}^{\prime}$ Elena (2003): Inacusatividad y aspecto léxico en los verbos de movimiento. Estudio diacrónico. Girona: Documenta Universitaria.

Corpas Pastor, Gloria (1997): Manual de fraseología española. Madrid: Gredos.

Corpas Pastor, Gloria (2003): Diez años de investigación en fraseología: análisis sintáctico-semánticos, contrastivos y dialectológicos. Madrid: Iberoamericana - Vervuert.

Covarrubias, Sebastián de (1611): Tesoro de la lengua castellana o española. Felipe C.R. Maldonado (ed.). Madrid: Castalia, 1995.

Galán Rodríguez, Carmen (1993): «Aproximación al estudio de los verbos de movimiento en alemán y en español: movimiento real y empleos figurados». En: Anuario de Estudios Filológicos, XVI, 147-158.

Koike, Kazumi (2001): Colocaciones léxicas en el español actual: estudio formal y léxico-semántico. Madrid: Universidad de Alcalá / Takushoku University.

García Jurado, Francisco (2000): «Las 'metáforas de la vida cotidiana' ('metaphors we live by') en latín y su proyección etimológica en castellano». En: Congreso Internacional de Semántica (Universidad de La Laguna, 1997). Madrid: Ediciones Clásicas, II, 1571-1584.

Lakoff, George y Mark Johnson (2001): Metáforas de la vida cotidiana. Madrid: Cátedra.

Lewis (2002): Lexical Approachs. Londres: Language Teaching Publications.

Mendívil Giró, José Luis (1999): Las palabras disgregadas. Sintaxis de las expresiones idiomáticas y los predicados complejos. Zaragoza: Prensas Universitarias de Zaragoza.

Ruiz Gurillo, Leonor (1998): La fraseología del español coloquial. Barcelona: Ariel.

Ruiz Gurillo, Leonor (2002): Ejercicios de fraseología. Madrid: Arco/Libros.

Ramos Alicia y Ana Serradilla (2000): Diccionario Akal del español coloquial. 1492 expresiones del español actual y más.... Madrid: Akal.

Serradilla Castaño, Ana (2001): «La enseñanza de frases hechas: un método para integrar la cultura en el aula». En: Actas del XI Congreso de ASELE. Zaragoza: Universidad, 657-664.

\section{GLAGOLA IR IN CAER KOT SESTAVNI DEL STALNIH BESEDNIH ZVEZ, KI NE IZRAŽAJO GIBANJA}

Glavni namen pričujočega prispevka je analiza španskih stalnih besednih zvez, sestavljenih iz glagolov gibanja (ir ali caer), ki kljub svojemu pomenu ne označujejo gibanja, ampak se nanašajo na psihološki proces ali na neko situacijo, ki s seboj ne prinaša nikakršnega realnega premikanja. Gre za oblike, kot so caer en saco roto, irse el santo al cielo o irse por los cerros de Úbeda. Avtorica na določenem korpusu analizira skladenjske in pomenoslovne značilnosti teh frazeologemov, ki se razlikujejo od kolokacij, in jih primerja s podobnimi strukturami v angleščini. Skuša ugotoviti, ali angleščina tudi uporablja glagole gibanja za izražanje enakih pomenov ali pa, nasprotno, uporablja glagole, ki označujejo stanje. Analizira vrednosti glagolov gibanja, ugotavlja vzroke rabe določenih metafor in poskuša odgovoriti na vprašanje, zakaj oba jezika v nekaterih primerih uporabljata glagole $\mathrm{z}$ metaforičnim pomenom za ustvarjanje tovrstnih izrazov, $\mathrm{v}$ drugih pa jih uporablja le španščina. 